\title{
Measurement of intrinsic top quark properties with the ATLAS detector
}

\author{
Lucia MASETTI ${ }^{* \dagger}$ \\ PRISMA Cluster of Excellence and Institute for Physics \\ Johannes Gutenberg University Mainz \\ E-mail: lucia.masetti@cern.ch
}

\begin{abstract}
Several measurements of intrinsic top quark properties performed with the ATLAS detector are presented: mass, charge, polarisation of the top quark and of the $W$ boson originating from its decay in order to test the structure of the $W t b$ vertex, as well as a search for top quark decays via flavour changing neutral currents. The results are based on up to $4.7 \mathrm{fb}^{-1}$ of data collected at the LHC in 2011 at a centre-of-mass energy of $7 \mathrm{TeV}$. Most measurements exploit the high statistics and low background level in the single lepton and dilepton channels and they require a full reconstruction of the event kinematics.
\end{abstract}

The European Physical Society Conference on High Energy Physics

18-24 July, 2013

Stockholm, Sweden

\footnotetext{
* Speaker.

On behalf of the ATLAS Collaboration
} 


\section{Introduction}

Already at the end of 2011 about one million pairs of top and antitop quarks ( $t \bar{t}$ events) had been produced at the LHC, the first collider that can be considered a top factory. The production cross-section could be measured [1] very early with the same level of precision as the theoretical prediction [2] and the large available statistics of top pair events can now be used to precisely test the properties of the top quarks. In spite of their smaller production cross-section and higher background contamination, single top quark events have also started recently to be used for properties measurements [3].

The measurements presented here are related to intrinsic properties of the top quark and of its decay: mass, charge, polarisation of the top quark and of the $W$ boson originating from a top quark decay and decay via Flavour Changing Neutral Currents (FCNC). These measurements are based on data collected by the ATLAS experiment [4] in 2011 at a centre-of-mass energy of $7 \mathrm{TeV}$ and corresponding to an integrated luminosity of up to $4.7 \mathrm{fb}^{-1}$.

The top quark decays to a $W$ boson and a $b$ quark with a branching fraction very close to $100 \%$. Hence, in a $t \bar{t}$ event the decay channels are determined by the decay modes of the $W$ boson. The single lepton and dilepton channels, with one or two $W$ 's decaying leptonically, respectively, are best suited for precision measurements of the top quark properties, thanks to their low level of background. In the following, only electrons and muons will be considered as charged leptons, since tau leptons would require special selection criteria. As a baseline for most analyses, events passing the single electron or single muon trigger were selected by requiring exactly one or exactly two oppositely charged isolated leptons with $|\eta|<2.5, p_{\mathrm{T}}>20 \mathrm{GeV}$ for muons and $E_{\mathrm{T}}>25$ $\mathrm{GeV}$ for electrons, missing transverse energy $\left(E_{\mathrm{T}}^{\text {miss }}\right)$ compatible with one or two neutrinos and at least 4 jets (single lepton) or at least 2 jets (dilepton) with $|\eta|<2.5, p_{\mathrm{T}}>25 \mathrm{GeV}$ and $75 \%$ of the scalar $p_{\mathrm{T}}$ sum of all the associated tracks originating from the primary vertex. In the single lepton channel at least one jet was required to be tagged as originating from a $b$ quark and the multijet background was reduced by applying further requirements on $E_{\mathrm{T}}^{\text {miss }}$ and on the transverse $W$ mass $m_{\mathrm{T}}^{\mathrm{W}}=\sqrt{2 p_{\mathrm{T}}^{\ell} E_{\mathrm{T}}^{\text {miss }}\left(1-\cos \left(\phi^{\ell}-\phi\left(E_{\mathrm{T}}^{\mathrm{miss}}\right)\right)\right.}$. In the dilepton channel the $Z+$ jets background was reduced by applying a veto on dilepton invariant masses around the nominal $Z$ mass for same flavour leptons or requiring the scalar $p_{\mathrm{T}}$ sum of all selected leptons and jets to be above $130 \mathrm{GeV}$ in the $e \mu$ channel.

The estimate of the background contamination is mostly based on Monte Carlo (MC) simulation, with the exception of fake lepton, $W+$ jets and $Z+$ jets contributions. The shape and normalisation of the fake lepton contribution originating from multijet events in the single lepton channel and from $W+$ jets events in the dilepton channel is determined from data using matrix methods. The normalisation of the $W+$ jets background in the single lepton channel is determined from data using the well predicted charge asymmetry in the $W$ production and the one of the $Z+$ jets background in the dilepton channel is determined from data in the tails of the dilepton invariant mass.

In order to associate jets with the quarks produced from the top quark decay a kinematic likelihood fit was used in the single lepton channel allowing for a full reconstruction of the event kinematics. A likelihood function containing Breit-Wigner distributions of the true parton or lepton energies around the nominal $W$ and $t$ mass, transfer functions taking into account the experimental resolution in the energy measurement and the $b$ tagging probability was maximised to choose the 


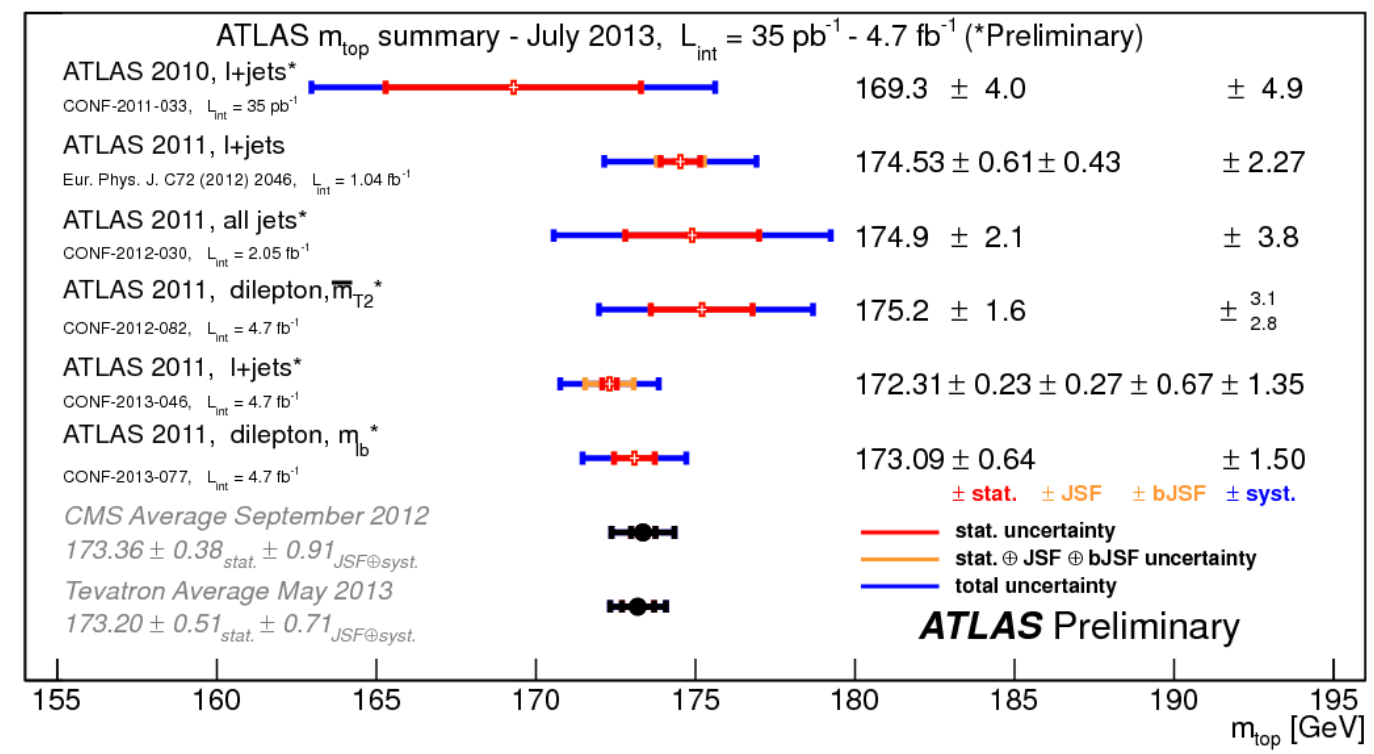

Figure 1: Summary of the ATLAS top mass measurements, compared to the CMS and Tevatron combinations [9].

best jet permutation, which was used for reconstruction. In the dilepton channel the momenta of the two neutrinos were inferred with different methods. As an example, the neutrino weighting method assigned a weight to each considered pair of neutrino pseudorapidities, depending on the compatibility of the total transverse momentum with the measured value of $E_{\mathrm{T}}^{\mathrm{miss}}$ [5].

Different categories of systematic uncertainties were considered: detector modelling uncertainties include the efficiency and resolution of object identification and reconstruction, the luminosity uncertainty and possible pile-up dependence; signal and background modelling uncertainties include the amount of QCD radiation, the choice of the MC generator model and of the Parton Distribution Functions (PDF), the uncertainty on the top quark mass, as well as the background normalisation and shape uncertainties and finally possible analysis specific uncertainties.

\section{Top quark mass and charge}

The measurements of the top quark mass preformed with the ATLAS experiment are summarised in Fig. 1, where they are compared with the CMS and Tevatron combined results [6, 7]. The latest measurements were performed in the single lepton [8] and dilepton [9] channels, based on $4.7 \mathrm{fb}^{-1}$ of data, yielding $m_{t}=172.31 \pm 0.23$ (stat) \pm 0.27 (JSF) \pm 0.67 (bJSF) \pm 1.35 (syst) and $m_{t}=173.09 \pm 0.64$ (stat) \pm 1.50 (syst), respectively. Their uncertainty is dominated by systematic effects, in particular in the single lepton channel by the uncertainty on the energy scale for all jets (JES) and the additional correction for jets originating from $b$ quarks. The effect on the top quark mass was reduced by simultaneously fitting a global scale factor for each and the uncertainty on the JES Scale Factor (JSF) and on the JSF for $b$ quarks (bJSF), quoted separately. More details on the measurement methods can be found in [10]. 

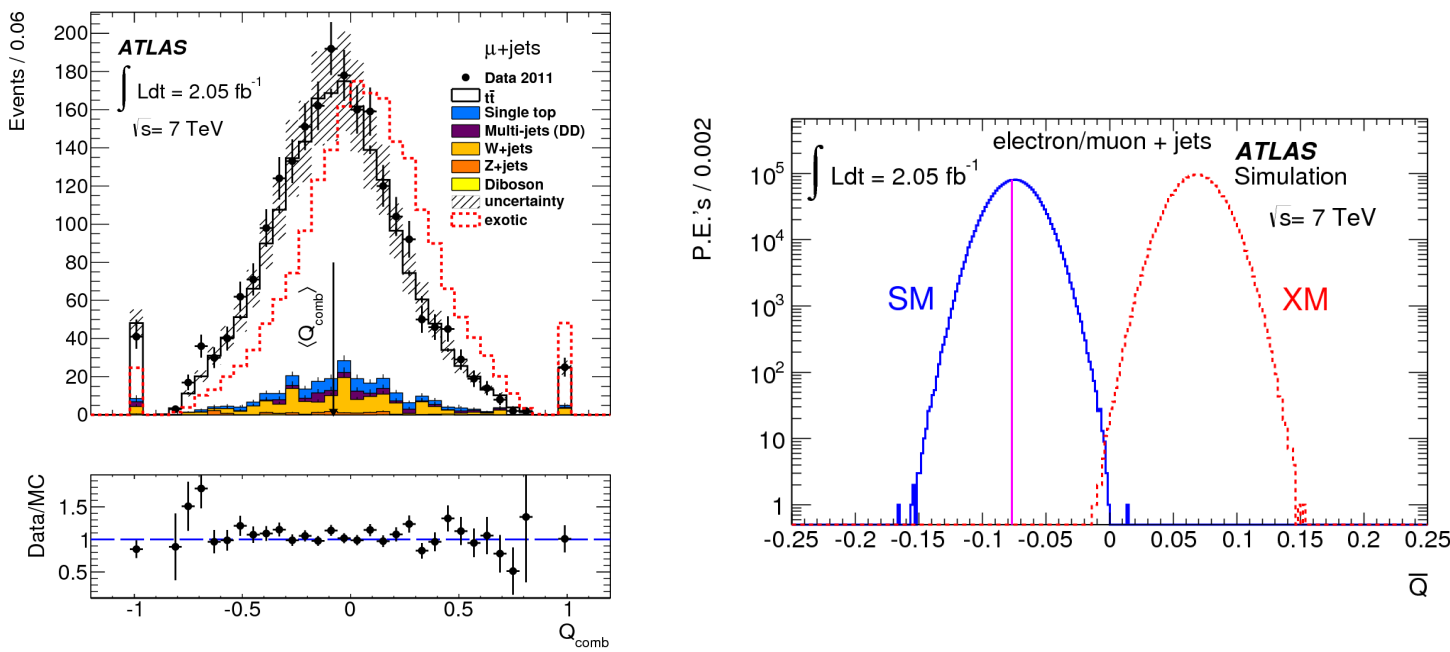

Figure 2: Left: $Q_{\text {comb }}$ distribution in the $\mu+$ jets channel for data, compared to the simulation of the Standard Model and of the exotic model. Right: Expected probability density functions of the $\bar{Q}$ variable under the Standard Model (SM) and eXotic Model (XM) hypotheses. The vertical line indicates the observed value [11].

To test whether the top quark observed experimentally is the particle predicted by the Standard Model with a charge of $2 / 3 e$ or an exotic particle with a charge of $-4 / 3 e$, the correlation between the charges of the $W$ boson and of the $b$ quark produced by the decay of a top quark was exploited in a measurement based on $2.05 \mathrm{fb}^{-1}$ of data in the single lepton channel with the additional requirement of exactly $2 b$-tagged jets [11]. The combined charge $Q_{\text {comb }}$ was computed as the product of the lepton charge and the charge of the $b$-tagged jet paired to the lepton (see Eq. 2.1). The paired $b$-tagged jet $\left(b_{\ell}\right)$ was chosen to be the one building the lower invariant mass with the lepton and only events fulfilling the requirements in Eq. 2.2 were used for the measurement to avoid confusion in the pairing.

$$
\begin{gathered}
Q_{\mathrm{comb}}=Q_{b_{\ell}} \cdot Q_{\ell}, Q_{b}=\frac{\sum_{i} Q_{i}\left|\vec{j} \cdot \vec{p}_{i}\right|^{0.5}}{\sum_{i}\left|\vec{j} \cdot \vec{p}_{i}\right|^{0.5}} \\
m_{\ell b_{1}}<m_{c r}, m_{\ell b_{2}}>m_{c r},
\end{gathered}
$$

with the weighted sum of charges running over a maximum number of ten tracks with $p_{\mathrm{T}}>1$ $\mathrm{GeV}$ associated with the $b$-tagged jet within a cone of $\Delta R<0.25$, the weights being the projection of the track momentum on the jet axis and $m_{c r}=155 \mathrm{GeV}$. The average value of the combined charge $\left(\left\langle Q_{\text {comb }}\right\rangle\right)$ measured in data was compared with the expectations for the SM and the exotic model obtained from pseudo-experiments, as shown in Fig. 2 (right). The measured value was found to be compatible with the Standard Model and the exotic model is excluded at a level of more than $8 \sigma$. Furthermore the value of the top quark charge was obtained from the background subtracted value of $\left\langle Q_{\text {comb }}\right\rangle$ using the $b$-jet charge calibration coefficient $C_{b}$ derived from $t \bar{t} \mathrm{MC}$ : $Q_{t}=1+C_{b} \cdot\left\langle Q_{\text {comb }}^{b k g-\text { sub }}>=0.64 \pm 0.02\right.$ (stat) \pm 0.08 (syst). 


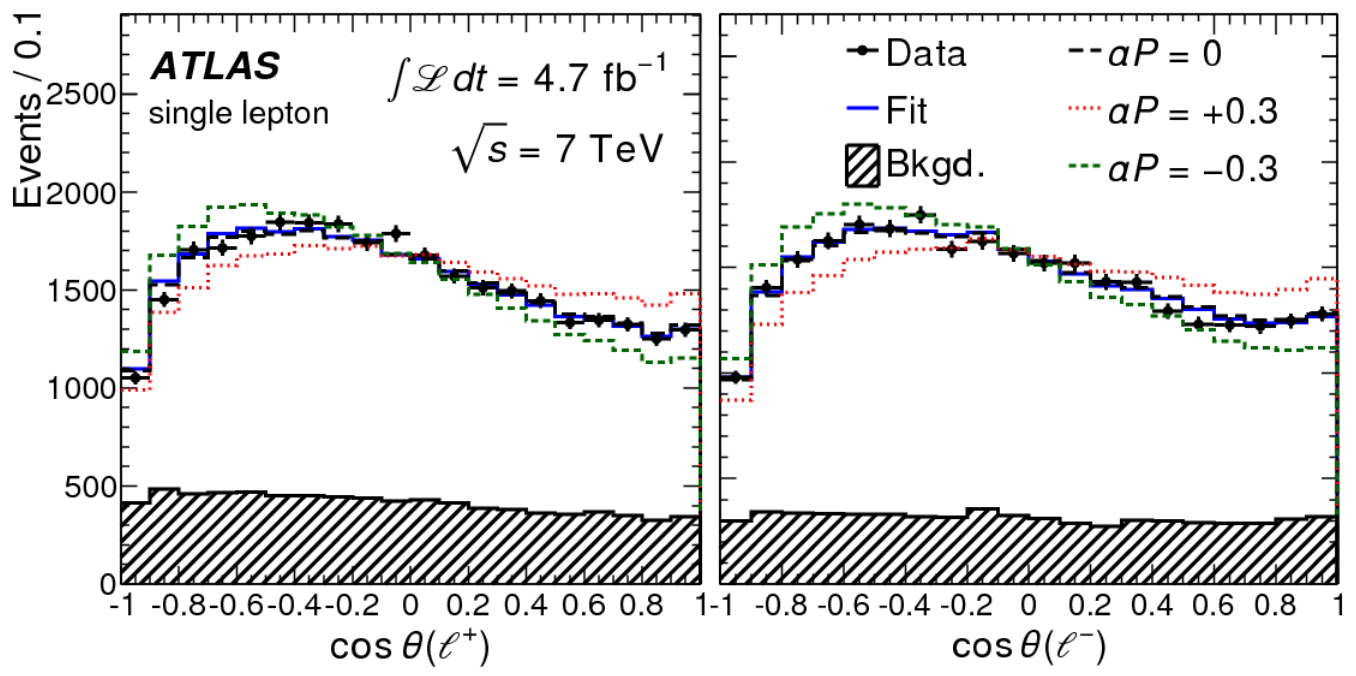

Figure 3: Template fit result of the top quark polarisation measurement in the single lepton channel for positively (left) and negatively (right) charged leptons assuming CP conservation [5].

\section{Polarisation of the top quark and of the $W$ boson from a top quark decay}

The polarisation of pair produced top quarks, predicted to be zero in the Standard Model, was measured in the single lepton and dilepton channels using $4.7 \mathrm{fb}^{-1}$ of data. Data were fitted to templates with different polarisation values in the $\cos \theta_{\ell}$ variable, where $\theta_{\ell}$ is the angle between the momenta of the charged lepton and of the top quark in the $t \bar{t}$ rest frame. Their distribution depends on the top quark polarisation $P$ and on the analysing power of the lepton $\alpha_{\ell}$ in the form $W\left(\cos \theta_{\ell}\right)=$ $1 / 2\left(1+\alpha_{\ell} P \cos \theta_{\ell}\right)$. From the template fit the fraction $f$ of positively polarised top quarks was measured separately under the hypotheses of CP conservation (see Fig. 3) or CP violation between top and antitop quark. Since the templates used in the fit had values of $\alpha_{\ell} P= \pm 0.3$, the polarisation was computed from $f$ as $\alpha_{\ell} P=0.6 f-0.3$. The obtained results

$$
\alpha_{\ell} P_{C P C}=-0.035 \pm 0.014 \text { (stat) } \pm 0.037 \text { (syst), } \alpha_{\ell} P_{C P V}=0.020 \pm 0.016(\text { stat })_{-0.017}^{+0.013} \text { (syst) }
$$

are in agreement with the SM prediction within the uncertainty under both considered hypotheses. The higher precision obtained under the $\mathrm{CP}$ violating hypothesis is due to the charge symmetric nature of most systematic variations.

The $W$ bosons originating from a top quark decay are predicted to be longitudinally polarised in about $70 \%$ of the cases and left-handed in the remaining ones, while the right-handed component is negligible. A precise measurement of the $W$ helicity fractions was performed in the single lepton and dilepton channels on $1.04 \mathrm{fb}^{-1}$ of data [12] using template fits of the longitudinal $\left(F_{0}\right)$, lefthanded $\left(F_{L}\right)$ and right-handed $\left(F_{R}\right)$ helicity fractions (see Eq. 3.2) and asymmetries $A_{ \pm}$(see Eq. 3.3) of the distribution in $\cos \theta^{*}$, where $\theta^{*}$ is the angle between the momenta of the charged lepton and of the top quark in the $\mathrm{W}$ rest frame.

$$
\begin{aligned}
\frac{\mathrm{d} \sigma}{\sigma \mathrm{d} \cos \theta^{*}} & =\frac{3}{4}\left(1-\cos ^{2} \theta^{*}\right) F_{0}+\frac{3}{8}\left(1-\cos \theta^{*}\right)^{2} F_{L}+\frac{3}{8}\left(1+\cos \theta^{*}\right)^{2} F_{R} \\
A_{ \pm} & =\frac{N\left(\cos \theta^{*}>z_{ \pm}\right)-N\left(\cos \theta^{*}<z_{ \pm}\right)}{N\left(\cos \theta^{*}>z_{ \pm}\right)+N\left(\cos \theta^{*}<z_{ \pm}\right)}
\end{aligned}
$$



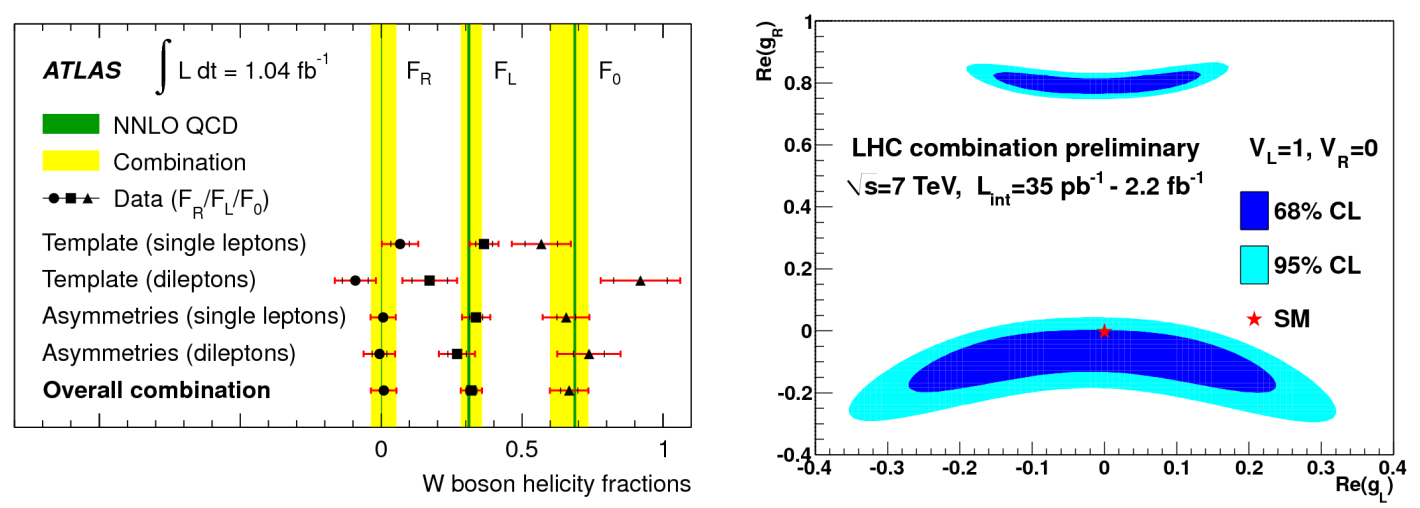

Figure 4: Left: Summary of the measured $\mathrm{W}$ helicity fractions using the template and asymmetry methods in the single lepton and dilepton channels. Their combination is compared to the Standard Model expectation [12]. Right: Allowed real parts of the anomalous couplings $g_{L}$ and $g_{R}$ based on the combination of the ATLAS and CMS measurements of the W helicity fractions [13].

with $z_{ \pm}= \pm\left(1-2^{2 / 3}\right)$, chosen to cancel the dependence on $F_{L}$ or $F_{R}$, respectively. The measured helicity fractions for each method and each channel, as well as their combination are shown in Fig. 4 (left). They all agree with the SM prediction within their respective uncertainties. A combination with similar CMS measurements required a detailed study of the correlations between the systematic uncertainties considered in each analysis and yielded $F_{0}=0.626 \pm 0.034$ (stat) \pm 0.048 (syst) and $F_{L}=0.359 \pm 0.021$ (stat) \pm 0.028 (syst) with the sum of the three fractions fixed to unity [13]. The results were interpreted in terms of allowed values of the anomalous couplings in the effective Lagrangian (see Eq. 3.4), as shown in Fig. 4 (right).

$$
\mathscr{L}_{W t b}=-\frac{g}{\sqrt{2}} \bar{b} \gamma^{\mu}\left(V_{L} P_{L}+V_{R} P_{R}\right) t W_{\mu}^{-}-\frac{g}{\sqrt{2}} \bar{b} \frac{i \sigma^{\mu v} q_{v}}{M_{W}}\left(g_{L} P_{L}+g_{R} P_{R}\right) t W_{\mu}^{-}+\text {h.c. }
$$

Fixing $V_{L}=1, V_{R}=g_{L}=0, \operatorname{Re}\left(g_{R}\right)$ was constrained to $-0.10 \pm 0.06(\text { stat })_{-0.08}^{+0.07}$ (syst).

\section{Search for FCNC decays}

A search for the FCNC decay $t \rightarrow Z q$, that is strongly suppressed in the Standard Model and can have a branching fraction as high as $10^{-4}$ in many models beyond it, was performed in the trilepton channel $(Z \rightarrow \ell \ell$ and the other top quark decaying to $W b$ with $W \rightarrow \ell v)$, based on $2 \mathrm{fb}^{-1}$ of data [14]. The object definitions were similar to the ones described in Section 1, but additionally to fully identified leptons also so called track leptons were used to increase the reconstruction efficiency of $\tau$ decays. The event selection and the background estimates were modified according to the different kinematics. No excess of events compatible with the searched decay was observed over the expected SM background and a limit on the FCNC branching fraction of $0.73 \%$ was set at 95\% confidence level.

\section{Conclusions}

Many precise measurements of top properties were performed using different decay channels, 
full kinematic reconstruction and data-driven estimates of the main background contaminations. All results confirm, within their uncertainties, the Standard Model predictions, in particular the ones concerning the top quark charge, the unpolarised pair production of top quarks and the Wtb vertex structure. The precision of the results is mostly limited by the systematic uncertainties. New methods to constrain systematic variations from data have been used successfully in particular in the top mass measurements.

\section{References}

[1] ATLAS Collaboration, Statistical combination of top quark pair production cross-section measurements using dilepton, single-lepton, and all-hadronic final states at $\sqrt{s}=7 \mathrm{TeV}$ with the ATLAS detector, ATLAS-CONF-2012-024, [http: //cds . cern. ch/record/1430733].

[2] M. Czakon, P. Fiedler, A. Mitov., The total top quark pair production cross-section at hadron colliders through $\mathscr{O}\left(\alpha_{S}^{4}\right)$, Phys. Rev. Lett. 110 (2013) 252004 [ 1303.6254 [hep-ph ] ] .

[3] A. Krasznahorkay, Single top quark production cross section in ATLAS, in proceedings of The European Physical Society Conference on High Energy Physics, POS (EPS-HEP 2013) 228.

[4] ATLAS Collaboration, The ATLAS Experiment at the CERN Large Hadron Collider, JINST 3 (2008) S08003

[5] ATLAS Collaboration, Measurement of top quark polarization in top-antitop events from proton-proton collisions at $\sqrt{s}=7 \mathrm{TeV}$ using the ATLAS detector, 1307.6511 [hep-ex ] .

[6] CMS Collaboration, Top mass combination, CMS-PAS-TOP-11-018, [http://cds.cern.ch/record/1478194].

[7] CDF and D0 Collaborations, Combination of CDF and DO results on the mass of the top quark using up to $8.7 \mathrm{fb}^{-1}$ at the Tevatron, 1305.3929 [hep-ex] .

[8] ATLAS Collaboration, Measurement of the Top Quark Mass from $\sqrt{s}=7$ TeV ATLAS Data using a 3-dimensional Template Fit, ATLAS-CONF-2013-046, [http://cds.cern.ch/record/1547327].

[9] ATLAS Collaboration, Measurement of the Top Quark Mass in Dileptonic Top Quark Pair Decays with $\sqrt{s}=7$ TeV ATLAS Data, ATLAS-CONF-2013-077,

[http://cds.cern.ch/record/1562935].

[10] G. Compostella, Top quark mass measurements in ATLAS, in proceedings of The European Physical Society Conference on High Energy Physics, POS (EPS-HEP 2013) 209.

[11] ATLAS Collaboration, Measurement of the top quark charge in pp collisions at $\sqrt{s}=7$ TeV with the ATLAS detector, 1307.4568 [hep-ex] .

[12] ATLAS Collaboration, Measurement of the W boson polarization in top quark decays with the ATLAS detector, JHEP 1206 (2012) 088 [1205.2484 [hep-ex] ] .

[13] ATLAS and CMS Collaborations, Combination of the ATLAS and CMS measurements of the W-boson polarization in top-quark decays, ATLAS-CONF-2013-033, [http://cds.cern. ch/record/1527531].

[14] ATLAS Collaboration, A search for flavour changing neutral currents in top-quark decays in pp collision data collected with the ATLAS detector at $\sqrt{s}=7$ TeV, JHEP 1209 (2012) 139 [1206.0257 [hep-ex]]. 